\title{
VICTOR: Vinflunine in advanced metastatic transitional cell carcinoma of the urothelium: A retrospective analysis of the use of vinflunine in multi-centre real life setting as second line chemotherapy through Free of Charge Programme for patients in the UK and Ireland
}

\author{
SYED A. HUSSAIN ${ }^{1,2}$, JAWAHER ANSARI $^{3}$, ROBERT HUDDART $^{4}$, DEREK G. POWER $^{5}$, JEANETTE LYONS $^{6}$, \\ JAMES WYLIE $^{6}$, MARIA VILARINO-VARLELA ${ }^{7}$, NILS O. ELANDER ${ }^{1,2}$, RHONA MCMENEMIN $^{8}$, \\ LISA M. PICKERING $^{9}$, GUY FAUST ${ }^{10}$, SEEMACHAUHAN ${ }^{1}$ and RICHARD J. JACKSON ${ }^{1}$ \\ ${ }^{1}$ University of Liverpool, Liverpool L69 3GA; ${ }^{2}$ The Clatterbridge Cancer Centre NHS Foundation Trust, Bebington, \\ Wirral CH63 4JY; ${ }^{3}$ Beatson West of Scotland Cancer Centre, Glasgow G12 0YN; ${ }^{4}$ Institute of Cancer Research and \\ Royal Marsden Hospital, Sutton, Surrey SM2 5NG, UK; ${ }^{5}$ The Mercy University Hospital, Wilton, Cork T12 WE28, \\ Republic of Ireland; ${ }^{6}$ The Christie NHS Foundation Trust, Manchester M20 4BX; ${ }^{7}$ Royal Free Hospital, \\ London NW3 2QG; ${ }^{8}$ Northern Centre for Cancer Care, Freeman Hospital, High Heaton, \\ Newcastle upon Tyne NE7 7DN; ${ }^{9}$ St George's Healthcare Trust, Tooting, London SW17 0QT; \\ ${ }^{10}$ Northampton General Hospital Trust, Cliftonville, Northampton NN1 5BD, UK
}

Received September 23, 2016; Accepted November 14, 2016

DOI: $10.3892 /$ ijo.2017.3847

\begin{abstract}
There is no standard of care in the UK or Ireland for second-line chemotherapy for patients with advanced transitional cell carcinoma (TCCU). Vinflunine is approved for TCCU patients who have failed a platinum-based regimen, and is standard of care in Europe but is not routinely available in the UK. Data were collected retrospectively on patients who received vinfluine as a second-line treatment. The aims were to document the toxicity and efficacy in a real life setting. Data were collected on 49 patients from 9 sites across the UK and Ireland [median age, 64 (IQR, 57-70) years, 33 males]. All patients had advanced metastatic TCCU. Thirteen patients had bone or liver metastases, 4 patients had PS 2 and 11 patients had $\mathrm{HB}<10$. Median vinflunine administration was 3.5 cycles (range 1-18). Most common grade 3-4 toxicities were constipation (4 patients) and fatigue (3 patients). Partial response rate was $29 \%$ (14 PR, 11 SD, 19 PD, 4 NE, 1 not available). Median OS was $9.1(6.0,12.7)$ months. Results are consistent with real life data from Europe. Toxicity is further reduced with prophylactic laxative and oral antibiotics. Vinflunine is an efficient and tolerable second line treatment in advanced TCCU.
\end{abstract}

Correspondence to: Dr Syed A. Hussain, Department of Molecular and Clinical Cancer Medicine, University of Liverpool, 5th Floor UCD, Duncan Building, Daulby Street, Liverpool L69 3GA, UK E-mail: syed.hussain@liverpool.ac.uk

Key words: vinflunine, TCCU, second line, overall survival

\section{Introduction}

Bladder cancer is a significant cause of morbidity and mortality in the UK with $\sim 10,000$ new cases diagnosed and 5,000 deaths registered each year (1). The most common pathological subtype is transitional cell carcinoma (TCCU) (90\% of cases) (2) with $\sim 50 \%$ of all cases being stage II or beyond at the time of diagnosis (1). Standard of care for localized, muscle invasive TCCU is radical cystectomy, which historically, i.e., without modern neoadjuvant combination chemotherapy, leads to 5-year overall survival in the approximate range of $50-60 \%$ (3).

A meta-analysis including 11 trials and 3,005 patients confirmed the value of preoperative cisplatin based combination chemotherapy which was shown to reduce the risk of relapsing disease and conferring a 5-year absolute overall survival advantage of $5 \%$ (4). A recent meta-analysis comprising 945 patients and nine clinical trials similarly demonstrated the benefits of postoperative cisplatin based combination chemotherapy (5). There is thus significant evidence supporting the use of chemotherapy in localized disease. However, not all patients are eligible for curative-intent multimodal therapy, and about one in five patients present with stage IV disease (i.e., locally extending to other organs, to the pelvic or abdominal wall, and/or with evidence of distant metastases) at the time of diagnosis (1).

Patients with relapse following primary treatment, or with advanced disease at presentation, confer a significant challenge, and even among those fit for optimal platinum-based combination chemotherapy the median overall survival does not exceed the range of $12-15$ months $(6,7)$. The recommended 
first line chemotherapy for these patients are cisplatin based combinations and either MVAC (methotrexate, vinblastine, doxorubicin, and cisplatin) or GC (gemcitabine and cisplatin) $(6,7)$ although the GC regimen is often preferred due to a milder toxicity profile (7). For patients with acceptable performance status and preserved organ functions, and where the relapse occurs later than 12 months following neoadjuvant/ adjuvant cisplatin-based combination chemotherapy, change of platinum based regimen may be a feasible option (8). In selected cases the addition of paclitaxel to gemcitabine and cisplatin may be considered (9). For patients unfit for cisplatin combinations alternative although potentially less efficient combination regimens have been proposed, either with alternative platinum agents [oxaliplatin (10) or carboplatin (11)] or a platinum-free combination of paclitaxel and gemcitabine (12). In patients deemed ineligible for standard cisplatin based treatment, combination treatment with split dose cisplatin and gemcitabine has reported encouraging results (13).

Following failure of first line chemotherapy, be it early relapse following platinum based neoadjuvant/adjuvant chemotherapy, or progressive disease during palliative firstline chemotherapy, treatment options have so far been limited. Studies, mostly phase II and retrospective series, have reported activity with taxanes and pemetrexed (14). Until recently there were no randomized studies to confirm the benefit of second line chemotherapy for patients with metastatic TCCU. Vinflunine (Javlor ${ }^{\circledR}$ ), a microtubule inhibitor of the vinca-alkaloid family of anticancer agents (15), is the first drug to obtain European Medicine Agency (EMA) approval for use in TCCU (2009) due to evidence of efficacy from phase II $(16,17)$ and phase III trials $(18,19)$. Considering the multiple challenges in the second-line setting, with declining performance status due to progressive disease, persistent side effects or complications from earlier treatments, and primary or acquired chemo resistance after primary chemotherapy, the safety profile and efficacy data from the recent vinflunine reports are encouraging. In the phase III trial $(18,19)$ median overall survival was 6.9 months in the vinflunine plus best supportive care compared to 4.3 months in the best supportive care only population.

Further empirical studies have confirmed vinflunine to be a safe and effective second line approach in Spain $[n=66$ (20)], France [n=134 (21)] and Germany [n=77, Hegele (22)] with reported overall survival of 7.7-10.4 months. Based on the accumulating evidence, the ESMO guidelines now suggest vinflunine as the recommended second-line therapy in advanced bladder cancer (23).

Vinflunine as a second line therapy is not currently recommended for UK practice, nor is it available to the NHS patients through the approved list of drugs on cancer drug fund. It has however been made available through the Free of Charge Program (FOCP) sponsored by Pierre Fabre. Here we evaluate the outcome of patients treated with vinflunine as a second line therapy in this program.

\section{Patients and methods}

Data were collected retrospectively on patients with advanced metastatic TCCU diagnosed between 6th June 1999 and 20th June 2013. Data were collected via a pre-defined CRF adopted for the study and sent to local investigators for population. All patients who received vinflunine as a second line therapy following failure of first line therapy were eligible for inclusion.

All patients received vinflunine as a second-line therapy through the free of charge program (FOCP) and received at least one dose of vinflunine. All patients were included in the analysis, irrespective of any dose reductions or toxicities. The dose of vinflunine was 320/280/ $250 \mathrm{mg} / \mathrm{m}^{2}$ every 3 weeks as per the SPc. The aim was to document the toxicity, radiological RR and OS for patients treated with vinflunine in real life setting within the FOCP.

Demographic data were collected on patient gender, age, height, weight and performance status as well as the site of the disease recurrence and their hemoglobin level at the time that the decision to prescribe vinflunine was made. Details on the type of first-line therapy were requested as was whether this was in the neo-adjuvant or adjuvant setting.

Continuous data are presented as medians (IQR) and categorical data are expressed as frequencies of counts. The primary outcome measure of interest is overall survival (OS) which is measured as the time from intention to treat with vinflunine until death by any cause. Survival estimates obtained via method of Kaplan and Meier. Progression-free survival (PFS) is measured as the time from first vinflunine administration until progression or death by any cause. Objective response rate is defined using RECIST criteria (version 1.1) and determined at each local site. Univariate analyses are carried out to assess patient demographics as prognostic factors for overall survival using Cox proportional hazards models. Hazard ratios (HR) are presented with associated $95 \%$ confidence intervals (CI). No multivariate analyses were carried out. All P-values are considered significant at the 5\% level. All analysis were carried out using R (version 3.1.2).

Reported toxicities are defined using CTCAE (version 4) and are reported based on being either grade 1-2 or 3-4, respectively.

\section{Results}

Data are provided on 49 patients from nine contributing sites throughout the UK and Ireland (CCC 15; Christie 5; Cork 6; Glasgow 9; St. George's 1; Royal Free London 2; Royal Marsden 6; Northampton 1; Newcastle 4) diagnosed with advanced metastatic TCCU between 6th June 1999 and 20th June 2013. All patients have progression of disease after first-line platinum based therapy. Patient demographics are provided in Table I. The patient group consists of 67\% (33/49) males and have a median (IQR) age of 64 (57-70). As per the summary of product characteristics (SPC) for vinflunine, patients with a $0 / 1$ performance status (ECOG) or a hemoglobin $(\mathrm{Hb})$ level $>10 \mathrm{~g} / \mathrm{dl}$ were targeted. Thirteen patients (27\%) had an ECOG performance status of 2 or a hemoglobin level $<10$. Sixteen patients $(33 \%)$ had either bone or liver recurrence. Two patients $(4 \%)$ had chronic constipation at presentation and two patients $(4 \%)$ had coronary artery disease.

Information on first-line therapy is available for 48/49 (98\%) of all participating patients. First line neo-adjuvant chemotherapy was given to $60 \%$ (27/48) and consisted of gemcitabine/ 
Table I. Patient characteristics.

\begin{tabular}{llc}
\hline Category & \multicolumn{1}{c}{ Level } & $\mathrm{n}=49$ \\
\hline Gender & Female & $16(33 \%)$ \\
& Male & $33(67 \%)$ \\
Weight, kg & Median (IQR) & $71(63-83)$ \\
Height, cm & Median (IQR) & $168(160-175)$ \\
Age, years & Median (IQR) & $64(57-70)$ \\
Performance & 0 & $12(26 \%)$ \\
status (n=46) & 1 & $30(65 \%)$ \\
& 2 & $4(9 \%)$ \\
Site of disease & Bone & $8(17 \%)$ \\
recurrence $(\mathrm{n}=46)$ & Liver & $11(24 \%)$ \\
& Lung & $15(33 \%)$ \\
& Other visceral & $8(17 \%)$ \\
& Other non-visceral & $3(7 \%)$ \\
Hemoglobin & None & $1(2 \%)$ \\
& $<10$ & $11(28 \%)$ \\
& $10-12$ & $20(50 \%)$ \\
& $>12$ & $9(22 \%)$ \\
& &
\end{tabular}

Table II. Vinflunine administration.

\begin{tabular}{lcc}
\hline Category & Level & \\
\hline Starting dose $\left(\mathrm{mg} / \mathrm{m}^{2}\right)$ & 250 & $2(4 \%)$ \\
& 280 & $37(75 \%)$ \\
& 320 & $10(21 \%)$ \\
Escalation $(\mathrm{n}=47)$ & No & $44(94 \%)$ \\
Reduction $(\mathrm{n}=47)$ & Yes & $3(6 \%)$ \\
& No & $37(79 \%)$ \\
Cycles & Yes & $10(21 \%)$ \\
\hline
\end{tabular}

cisplatin (74\%; 20/27), gemcitabine/carboplatin $(22 \%, 6 / 27)$, or gemcitabine/cisplatin switched to carboplatin in view of worsening renal functions $(4 \%, 1 / 27)$. In the metastatic setting patients received platinum regimens consisting of either gemcitabine/cisplatin $(30 \%, 13 / 43)$, gemcitabine/carboplatin $(37 \%, 16 / 43)$, gemcitabine/cisplatin switched to carboplatin (2\%, 1/43), or some other combination $(30 \%, 13 / 43)$.

Following first-line therapy, patient best response rates were complete response $15 \%$ (7/48), partial response $48 \%(23 / 48)$, stable disease $17 \%(8 / 48)$ and progressive disease $19 \%(9 / 48)$. Best response was not available for one patient. The overall response rate for first-line therapy was 63\% (30/48) and $98 \%$ (43/44) of patients demonstrated disease progression with $77 \%$ (34/44) demonstrating bone, liver, or lung metastases.
Table III. Reported hematological and non-hematological toxicities.

\begin{tabular}{lrc}
\hline & \multicolumn{2}{c}{ Grade } \\
\cline { 2 - 3 } Toxicity & $1-2$ & $3-4$ \\
\hline Hematological & & \\
Anemia & 5 & 2 \\
Neutropenia & 13 & 1 \\
Neutropenic infection & 0 & 2 \\
Thrombocytopenia & 2 & 0 \\
Febrile infection & 1 & 0 \\
Leukopenia & 1 & 1 \\
Other & 0 & 2 \\
Non-hematological & & \\
Constipation & 9 & 3 \\
Asthenia/fatigue & 20 & 0 \\
Vomiting & 8 & 0 \\
Abdominal pain & 2 & 2 \\
Other & 0 &
\end{tabular}

Table II gives the details of vinflunine administrations. At the point of data collection 248 cycles of vinflunine had been administered. The starting dose was $250 \mathrm{mg} / \mathrm{m}^{2}$ (4\%, 2/49), $280 \mathrm{mg} / \mathrm{m}^{2}(76 \%, 37 / 49)$ or $320 \mathrm{mg} / \mathrm{m}^{2}$ (20\%, 10/49). Three patients $(6 \%)$ had a dose escalation and $10(21 \%)$ had dose reduction. The median (IQR) number of cycles given was 3.5 (2-6.25). Eight patients (17\%) had only a single cycle, while the maximum number of cycles at the time of analysis was 18 .

Toxicity. Toxicities are reported by sites as being either grade $1 / 2$ or grade $3 / 4$ hematological or non-hematological. Results are given in Table III. Overall 31\% (15/49) of patients reported at least one grade $3 / 4$ adverse event. The most common grade $3 / 4$ toxicities are constipation reported by $8 \%(4 / 49)$ and asthenia/fatigue reported by $6 \%(3 / 49)$ of patients.

Overall survival. Patients were followed up for a median of 9.1 months. At the time of analysis, $41(84 \%)$ patients had died. Overall survival estimates obtained via the method of Kaplan-Meier (Fig. 1) give a median (95\% confidence interval) of 9.1 months (6.0-12.7). ECOG performance measures were investigated further as a key prognostic indicator of interest (Fig. 2). Median survival estimates of $13.1(6.0, N A)$ months and 7.6 (5.98-10.5) months were observed for ECOG performance measures of 0 and 1-2, respectively. This was not significant statistically [HR (95\% CI): $1.62(0.74,3.55) ; \mathrm{P}=0.23$ ].

Disease progression. A total of 45 (92\%) of patients had disease progression at the time of analysis. Median progression-free survival estimates (Fig. 2) are 5.1 months (4.3-8.7).

Patient response. Forty-eight patients were evaluable for overall response (Table IV). One patient did not provide any response data. 


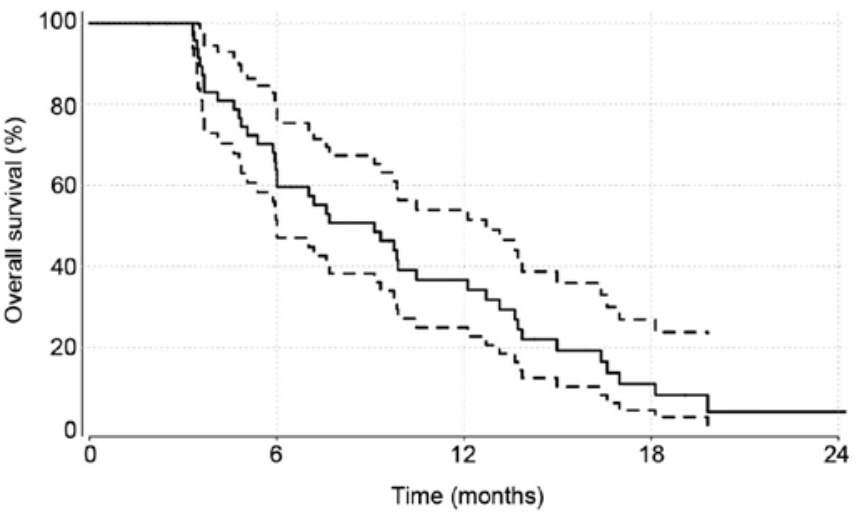

Numbers at risk

Tot 49

30

15

4

Figure 1. Kaplan-Meier plot of overall survival.

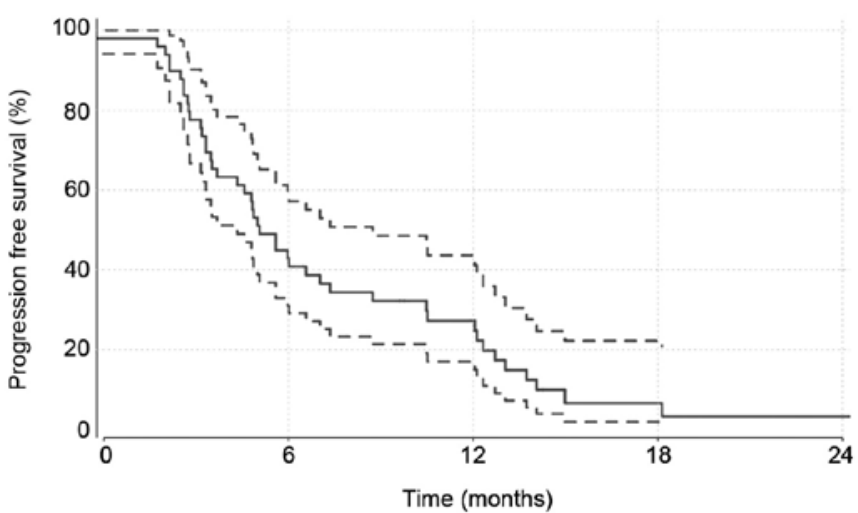

Numbers at risk

Tot 48

21

11

2

Figure 2. Kaplan-Meier plot for progression-free survival.

Table IV. Overall best response for vinflunine administration.

\begin{tabular}{lc}
\hline \multicolumn{3}{c}{ Best response } \\
\hline CR & 0 \\
PR & $14(29 \%)$ \\
SD & $10(21 \%)$ \\
PD & $19(40 \%)$ \\
NE & $4(8 \%)$ \\
\hline
\end{tabular}

Sensitivity analyses report the result only on patients who meet the SPC $($ ECOG $<2, \mathrm{HB}>10)$. Here 35 patients gave an estimated OS of 9.9 months (7.6-16.4) with an estimated PFS of 6.0 months (4.8-12.1) and a partial response rate of $30 \%$.

\section{Discussion}

The prognosis for patients with advanced metastatic TCCU who have progressed after first-line therapy remains dismal, and whilst previously published randomised trials and empirical evidence have favoured the use of vinflunine in this patient group, the results have not been clear enough to favour its wide spread use in the UK. As a consequence, many patients will end antitumour therapy or proceed onto palliative care once the first-line regimen has failed.

We performed a retrospective observational study in this clinical setting to assess the effects of vinflunine in terms of both efficacy and toxicity. Data on 49 patients from sites across the UK and Ireland were collected and analysed. The dose of and number of cycles were consistent with previous studies, with a median of 3.5 cycles being similar to the number recorded in the phase III trial $(18,19)$. The most common starting dose was $280 \mathrm{~g} / \mathrm{m}^{2}(75 \%)$ and the dose was relatively well tolerated with only $10(29 \%)$ patients requiring a reduction. There was no evidence of any correlation between the starting dose and dose reduction.

Further to this, the present median overall survival of 9.1 months (6.0-12.7) is consistent with or even slightly better than the 6.9 months of the vinflunine treated arm of the Bellmunt phase III study $(18,19)$. Further, the present numbers compare favourably with the empirical 'real life' results obtained from Spain (16) Germany (22) and France (21) which all report median survivals between 7.7-10.4 months in similar patient populations.

Toxicity rates observed in our study also show little cause for concern with $39 \%$ (19/49) patients reporting a grade $3+$ adverse event, the most common being constipation (4 patients) and fatigue ( 3 patients). Toxicity can be further improved with the use of prophylactic laxative and oral antibiotics and should provide little concern in the prescribing of vinflunine.

Further inspection of study efficacy shows that patients with a baseline ECOG PS of 0 display a median OS of 13.1 (6.0, NA) months, while patients of PS 1-2 have a poorer prognosis of $7.6(6.0-10.5)$ months. This is consistent with the data of ref. 20 reporting survival estimates of 13.2 and 6.7 months, respectively, for the same patient groups and emphasise baseline PS as a key prognostic predictor although not ruling out the potential benefits even in the PS 1-2 population.

This study is limited by the retrospective nature of the data collection and the lack of a randomised comparative arm against which to compare the efficacy, which ultimately open the study results to possible bias. In saying that, however, the study analysis includes patients who did not meet the SPC for vinflunine as well as patients who only received a single dose. Coupled with the high rate of ECOG $>0$ and the distribution of metastases this suggests a patient population which is representative of general clinical practice rather than a narrowly selected clinical trial population. Recently there has been much interest in the role of immune checkpoint inhibitors in urothelial cancer with durable responses have been noted with PD1 [e.g., pembrolizumab (24) and PDL1 atezolizumab (25)]. The results of recently completed trials comparing anti-PD1/PDL1 antibodies, with standard of care chemotherapy of institution's choice that includes vinflunine in the 2nd line setting (NCT 02256436) are awaited with interest.

In conclusion, the empirical results obtained from UK and Ireland practice reflect what has been observed in randomised trials of vinflunine as well as other retrospective studies 
throughout the European Union. The accumulating evidence puts forward vinflunine single drug therapy as an efficient, safe and reasonably tolerable second-line therapy in patients with advanced TCCU.

\section{References}

1. Cancer Research UK: Statistics 2012-2013. http://www.cancer researchuk.org/health-professional/cancer-statistics/statistics-bycancer-type/bladder-cancer.

2. Amin MB, McKenney JK, Paner GP, Hansel DE, Grignon DJ, Montironi R, Lin O, Jorda M, Jenkins LC, Soloway M, et al; International Consultation on Urologic Disease-European Association of Urology Consultation on Bladder Cancer 2012 . ICUD-EAU international consultation on bladder cancer 2012: Pathology. Eur Urol 63: 16-35, 2013.

3. Madersbacher S, Hochreiter W, Burkhard F, Thalmann GN, Danuser H, Markwalder R and Studer UE: Radical cystectomy for bladder cancer today -a homogeneous series without neoadjuvant therapy. J Clin Oncol 21: 690-696, 2003.

4. Advanced Bladder Cancer Meta-analysis Collaboration: Neoadjuvant chemotherapy for invasive bladder cancer. Cochrane Database Syst Rev: 1, 2004.

5. Leow JJ, Martin-Doyle W, Rajagopal PS, Patel CG, Anderson EM, Rothman AT, Cote RJ, Urun Y, Chang SL, Choueiri TK, et al: Adjuvant chemotherapy for invasive bladder cancer: A 2013 updated systematic review and meta-analysis of randomized trials. Eur Urol 66: 42-54, 2014.

6. Loehrer PJ Sr, Einhorn LH, Elson PJ, Crawford ED, Kuebler P, Tannock I, Raghavan D, Stuart-Harris R, Sarosdy MF, Lowe BA, et al: A randomized comparison of cisplatin alone or in combination with methotrexate, vinblastine, and doxorubicin in patients with metastatic urothelial carcinoma: A cooperative group study. J Clin Oncol 10: 1066-1073, 1992.

7. von der Maase H, Hansen SW, Roberts JT, Dogliotti L, Oliver T, Moore MJ, Bodrogi I, Albers P, Knuth A, Lippert CM, et al: Gemcitabine and cisplatin versus methotrexate, vinblastine, doxorubicin, and cisplatin in advanced or metastatic bladder cancer: Results of a large, randomized, multinational, multicenter, phase III study. J Clin Oncol 18: 3068-3077, 2000.

8. Necchi A, Pond GR, Giannatempo P, Di Lorenzo G, Eigl BJ, Locke J, Pal SK, Agarwal N, Poole A, Vaishampayan UN, et al: Cisplatin-based first-line therapy for advanced urothelial carcinoma after previous perioperative cisplatin-based therapy. Clin Genitourin Cancer 13: 178-184, 2015.

9. Bellmunt J, von der Maase H, Mead GM, Skoneczna I, De Santis M, Daugaard G, Boehle A, Chevreau C, Paz-Ares L, Laufman LR, et al: Randomized phase III study comparing paclitaxel/cisplatin/gemcitabine and gemcitabine/cisplatin in patients with locally advanced or metastatic urothelial cancer without prior systemic therapy: EORTC Intergroup Study 30987. J Clin Oncol 30: 1107-1113, 2012.

10. Carles J, Esteban E, Climent M, Font A, Gonzalez-Larriba JL, Berrocal A, Garcia-Ribas I, Marfa X, Fabregat X, Albanell J, et al; Spanish Oncology Genito Urinary Group Study Group: Gemcitabine and oxaliplatin combination: A multicenter phase II trial in unfit patients with locally advanced or metastatic urothelial cancer. Ann Oncol 18: 1359-1362, 2007.

11. De Santis M, Bellmunt J, Mead G, Kerst JM, Leahy M, Maroto P Skoneczna I, Marreaud S, de Wit R and Sylvester R: Randomized phase II/III trial assessing gemcitabine/ carboplatin and methotrexate/carboplatin/vinblastine in patients with advanced urothelial cancer 'unfit' for cisplatin-based chemotherapy: Phase II -results of EORTC study 30986. J Clin Oncol 27: 5634-5639, 2009.

12. Calabrò F, Lorusso V, Rosati G, Manzione L, Frassineti L, Sava T, Di Paula ED, Alonso S and Sternberg CN: Gemcitabine and paclitaxel every 2 weeks in patients with previously untreated urothelial carcinoma. Cancer 115: 2652-2659, 2009.
13. Hussain SA, Stocken DD, Riley P, Palmer DH, Peake DR, Geh JI, Spooner D and James ND: A phase I/II study of gemcitabine and fractionated cisplatin in an outpatient setting using a 21-day schedule in patients with advanced and metastatic bladder cancer. Br J Cancer 91: 844-849, 2004

14. Bambury RM, Benjamin DJ, Chaim JL, Zabor EC, Sullivan J, Garcia-Grossman IR, Regazzi AM, Ostrovnaya I, Apollo A, $\mathrm{Xiao} \mathrm{H}$ and Voss: The safety and efficacy of single-agent pemetrexed in platinum-resistant advanced urothelial carcinoma: A large single-institution experience. Oncologist 20: 508-515, 2015.

15. Bennouna J, Delord JP, Campone M and Nguyen L: Vinflunine: A new microtubule inhibitor agent. Clin Cancer Res 14: 1625-1632, 2008.

16. Culine S, Theodore C, De Santis M, Bui B, Demkow T, Lorenz J, Rolland F, Delgado FM, Longerey B and James N: A phase II study of vinflunine in bladder cancer patients progressing after first-line platinum-containing regimen. Br J Cancer 94: 1395-1401, 2006

17. Vaughn DJ, Srinivas S, Stadler WM, Pili R, Petrylak D, Sternberg CN, Smith DC, Ringuette S, de Wit E, Pautret V, et al: Vinflunine in platinum-pretreated patients with locally advanced or metastatic urothelial carcinoma: Results of a large phase 2 study. Cancer 115: 4110-4117, 2009.

18. Bellmunt J, Théodore C, Demkov T, Komyakov B, Sengelov L, Daugaard G, Caty A, Carles J, Jagiello-Gruszfeld A, Karyakin O, et al: Phase III trial of vinflunine plus best supportive care compared with best supportive care alone after a platinumcontaining regimen in patients with advanced transitional cell carcinoma of the urothelial tract. J Clin Oncol 27: 4454-4461, 2009.

19. Bellmunt J, Fougeray R, Rosenberg JE, von der Maase H, Schutz FA, Salhi Y, Culine S and Choueiri TK: Long-term survival results of a randomized phase III trial of vinflunine plus best supportive care versus best supportive care alone in advanced urothelial carcinoma patients after failure of platinumbased chemotherapy. Ann Oncol 24: 1466-1472, 2013.

20. Castellano D, Puente J, de Velasco G, Chirivella I, LópezCriado P, Mohedano N, Fernández O, García-Carbonero I, González MB and Grande E: Safety and effectiveness of vinflunine in patients with metastatic transitional cell carcinoma of the urothelial tract after failure of one platinum-based systemic therapy in clinical practice. BMC Cancer 14: 779, 2014.

21. Medioni J, Guillot A, Spaeth D, Di Palma M and Theodore C: Historical data in real life from patients treated by vinflunine for an advanced or metastatic urothelial carcinoma: Results of the CURVE study. Eur J Cancer 49: S646-S647, 2013.

22. Hegele A, De Geeter P, Goebell P, Matz U, De Schultz W and Retz M: Vinflunine in routine practice for the treatment of advanced or metastatic urothelial cell carcinoma in Germany. Eur J Cancer 49: 669-669, 2013.

23. Bellmunt J, Orsola A, Maldonado X and Kataja V; ESMO Guidelines Working Group: Bladder cancer: ESMO Practice Guidelines for diagnosis, treatment and follow-up. Ann Oncol 21 (Suppl 5): v134-v136, 2010.

24. Plimack ER, Bellmunt J, Gupta S, Berger R, Montgomery RB, Heath K, Juco J, Emancipator K, Pathiraja K, Lunceford JK, et al: Pembrolizumab (MK-3475) for advanced urothelial cancer: Updated results and biomarker analysis from. J Clin Oncol 33: 4502, 2015.

25. Hoffman-Censits JH, Grivas P, Van Der Heijden MS, Dreicer R Loriot Y, Retz M, Vogelzang NJ, Perez-Gracia JL, Rezazadeh A, Bracarda S, et al: IMvigor 210, a phase II trial of atezolizumab (MPDL3280A) in platinum-treated locally advanced or metastatic urothelial carcinoma (mUC). Genitourinary cancer meeting: 2016 Genitourinary cancers symposium. Welcome and general session 4: Immunotherapy for urothelial carcinoma. J Clin Oncol 34 (Suppl 2): 355, 2016. 